\title{
LV-IL-2
}

\section{The future of liver transplantation: Ex-vivo machine perfusion}

\author{
Pierre-Alain CLAVIEN*
}

Department of Surgery and Transplantation, University Hospital Zurich, Zurich, Switzerland

Lecture: Long-term preservation of liver grafts outside of the body (ex vivo) may enable treatment, repair, assessment of poor-quality livers currently declined for transplantation and regeneration of partial livers, thereby mitigating global donated liver shortage. The challenge, however, is to keep injured or resected human livers viable for multiple days, a time window necessary for expanded therapeutic interventions. Here, we present a liver perfusion technology that maintains ex vivo human liver function for seven days. The perfusion apparatus was engineered recapitulating core endocrine, renal, pulmonary, cardiac, diaphragmatic, and other physiologic functions for the liver, which are prerequisite for successful long-term liver preservation. The machine was developed in a stepwise fashion using swine livers and eventually tested in injured human livers declined for transplantation and in partial livers from patients, who have undergone formal hepatectomy. Perfused human livers, discarded for poor quality and predicted failure after transplantation, as well as the partial livers unveiled preserved function during ex vivo machine perfusion, as indicated by bile production, synthesis of coagulation factors and maintained cellular energy, among many others. Histological examinations of serial biopsies confirmed an intact liver structure at day seven. This data demonstrates, first, that injured human livers may recover from lethal injury and maintain function for one week outside of the body and second, that the availability of this machine perfusion system may provide an important tool for assessment of liver quality, to induce liver regeneration in partial livers, and to perform therapeutic interventions prior to transplantation. 